\title{
ARTICLE
}

\section{Medication management following first-episode psychosis in young people: a developmental approach}

\author{
Jo Richards
}

\begin{abstract}
Jo Richards is a consultant psychiatrist who works in an early intervention in psychosis team in Buckinghamshire. She is interested in using developmental concepts to enhance psychiatric clinical practice. She has worked as a consultant psychiatrist within child and adolescent and early intervention services and is concerned with promoting mental health service provision for young people which crosses conventional age barriers. Correspondence Dr Jo Richards, Buckinghamshire Early Intervention Team, Whiteleaf Wellbeing and Mental Health Centre, Aylesbury HP19 8JR, UK. Email: Josephine. Richards@oxfordhealth.nhs.uk
\end{abstract}

†For a discussion of antibodymediated encephalitis in Advances see Rickards H, Jacob S, Lennox B, et al (2014) Autoimmune encephalitis: a potentially treatable cause of mental disorder. Advances in Psychiatric Treatment, 20 92-100. Ed.

\section{SUMMARY}

Young adults and adolescents who experience a first episode of psychosis are faced with important issues and choices concerning their future mental health. Individual and family psychological treatments and psychosocial supportive measures are now well recognised interventions for this group of individuals. This article considers the complexity surrounding medication management after the initial recovery from psychosis. The discussion focuses particularly on the medical treatments, developmental considerations and roles of psychiatrists in improving outcomes for this group of patients.

\section{LEARNING OBJECTIVES}

- Understand the potential benefits and risks of maintenance antipsychotics for young people recovering from first-episode psychosis.

- Have an improved knowledge of psychological and developmental concepts that can underpin young people's decision-making regarding maintenance medication for mental illness.

- Be able to develop a range of (age-appropriate) strategies to assist medication decision-making.

\section{DECLARATION OF INTEREST}

None.

Individuals experiencing a first episode of psychosis usually respond well to both firstand second-generation (typical and atypical) antipsychotics. The evidence primarily relates to those over 18 years of age, and in reviewing it for their own study, Josiassen et al (2010) found that low doses are generally sufficient. They describe rapid symptom reduction following treatment with second-generation antipsychotics among their own sample of 60 individuals aged 18 to 30 with severe psychopathology. For those who fail to respond adequately to a course of two antipsychotics, the important role of clozapine is well recognised.

There is limited evidence supporting medication treatment for children and adolescents with psychoses, including schizophrenia (Kennedy 2007). Nevertheless, the recent National Institute for Health and Care Excellence (NICE) guidelines for this younger age group recommend that antipsychotic treatment be offered, including clozapine for treatment resistance (National Institute for Health and Clinical Excellence 2013). The role of autoantibodies for a subgroup of individuals with first-episode psychosis who show poor response to antipsychotic treatment (Lennox 2012) reminds us of the many unanswered questions about causes of psychotic disorders as a whole and the need for further research to define subgroups. ${ }^{\dagger}$

\section{Early intervention in psychosis teams}

This article focuses on how to build on recovery from a first episode of psychosis following treatment with antipsychotics. Early intervention in psychosis (EIP) teams provide an intense and comprehensive treatment package to aid recovery and they are recommended in NICE guidelines (National Institute for Health and Clinical Excellence 2013; National Institute for Health and Care Excellence 2014). Designed for young people during the early stages of mental illness, these teams smooth the transition from child and adolescent to adult mental health services. They generally provide treatment for individuals aged 14-35 years, although this can vary. This service model has a strong focus on the needs of young people more generally as they negotiate the path into adulthood (Box 1).

Individuals are referred to EIP teams on the basis of psychotic symptoms and other threshold criteria. There are a number of differential and comorbid diagnoses to consider, in addition to schizophrenia, as the clinical picture emerges over time (Box 2). Although some have argued against allocating resources to EIP teams, there is evidence to support their effectiveness, particularly during their engagement in an individual's treatment (Marshall 2011). Patient satisfaction with the EIP approach is also high (Lester 2011). 
BOX 1 Features of early intervention in psychosis (EIP) services

- They straddle the traditional boundary between child and adolescent and adult mental health services

- Patients are usually between 14 and 35 years old

- Each care coordinator has a relatively low number of patients

- Diagnostic uncertainty is embraced

- An active outreach approach to engagement is taken

- A range of individual and family treatments are offered

- Low doses of antipsychotic medication are used where possible

More research is needed to determine the most effective ingredients of the EIP approach. It seems likely that one of the most important components is active engagement. Following engagement, individuals can make use of a range of psychosocial and medical interventions that are likely to be effective in preventing relapse of psychosis and improving outcomes more generally. However, the art of engagement is subtle. The findings of the UK National Evaluation of the Development and Impact of Early Intervention Services (EDEN) group highlight how overzealous approaches by clinicians can be experienced as intrusive by young patients (Lester 2011).

EIP teams usually work with adolescents as well as young adults because most (but not all) psychotic disorders have their onset at this stage in life (Jones 2013). Despite being most at risk for a range of mental disorders, young people are the least likely to use services (Gulliver 2010).

B0X 2 Differential and comorbid diagnoses following first-episode psychosis

The following differential and comorbid ICD-10 diagnoses should be considered after a first psychotic episode:

- Schizophrenia, schizotypal and delusional disorders (F20-F29)

- Mood (affective) disorders (F30-F39)

- Mental and behavioural disorders due to psychoactive substance use (F10-19)

- Reaction to severe stress, and adjustment disorders (F43)

- Dissociative (conversion) disorders (F44)

- Disorders of adult personality and behaviour (F60-F69)

- Pervasive developmental disorders (F84)

(World Health Organization 1992)
Hence, the importance of a flexible engagement approach, which takes account of age-appropriate developmental and social factors. Popular communication technologies can provide a youthfriendly format for disseminating information about mental health. Such resources are likely to be most useful if linked to actual local youth and mental health services.

Medication strategies following recovery from first-episode psychosis may be seen as relatively straightforward and easy to access compared with psychological treatments. Further examination, however, reveals the complexity that surrounds this aspect of treatment. A recent survey of staff in an EIP team provides us with a starting point for the discussion of maintenance medication: all the staff surveyed found it 'difficult' or 'very difficult' to persuade patients to stay on medication once positive symptoms had receded (details available from the author on request).

\section{Risk of relapse and role of maintenance antipsychotics}

\section{Maintenance medication}

NICE guidelines highlight the high risk of relapse if antipsychotic medication is stopped following recovery from a first episode of psychosis or schizophrenia, and the necessity of considering each patient's needs and preferences individually (National Institute for Health and Care Excellence 2014). For those under 18, there is a lack of long-term follow-up studies to guide practice. Nevertheless, NICE advises against intermittent symptom-targeted medication (see below) as a routine approach following recovery (National Institute for Health and Clinical Excellence 2013; National Institute for Health and Care Excellence 2014).

There are difficulties comparing studies that have examined the risk of relapse after first-episode psychosis. For example, definitions of relapse and the availability of non-biological treatments vary in different studies. Diagnostic terminology is also a problem. Some of the more recent research has used the broader definition of first-episode psychosis, whereas earlier studies tended to focus on schizophrenia. Use of this broader definition is in keeping with the aforementioned EIP service delivery model adopted in many countries over the past 20 years, including the UK.

The most recent systematic reviews (AlvarezJimenez 2009; Leucht 2012) concluded that maintenance antipsychotic treatment reduces relapse after both first-episode schizophrenia and first-episode psychosis. However, none of the studies reviewed characterise the subgroup 
of individuals who can successfully discontinue medication. Individual studies will also be of keen interest to patients and clinicians. Longer-term follow-up studies have indicated relatively high rates of relapse even on maintenance medication. A 5-year follow-up of 118 patients with firstepisode schizophrenia or schizoaffective disorder found that relapse rates were high overall, at $80 \%$, and increased by a factor of five in those who discontinued antipsychotic treatment (Robinson 1999). More recent follow-up studies have generally indicated significantly increased rates of relapse for individuals who discontinue antipsychotics or significantly reduce their dose compared with those who stay on maintenance treatment.

For example, a Hong Kong group have reported a controlled trial in which 178 individuals with first-episode psychosis treated with quetiapine for 1 year were subsequently randomised equally to quetiapine or to placebo (Chen 2010). The estimated risk of relapse at 12-month follow-up was $41 \%$ for the quetiapine group and $79 \%$ for the placebo group.

In contrast, a Dutch group reporting on a 7-year follow-up of 103 people with first-episode psychosis found no significant difference in the rates of relapse for both groups (Wunderink 2013). However, those who discontinued medication or significantly reduced the dose had better recovery than the maintenance treatment group ( $40 \%$ v. $17.5 \%)$. It should be noted that recovery was restrictively defined as symptomatic and functional remission for only the preceding 6 months at the time of the follow-up. Furthermore, the overall number of participants who had recovered was small (21 who had discontinued or significantly reduced medication $v .9$ on maintenance treatment). The authors themselves point to the need for replication of their findings before change of practice can be recommended. In a related commentary, McGorry et al (2103) argue for further discussion on agreeing relevant outcome measures rather than focusing on relapse rates alone. Nevertheless, relapse is a significant life disruption for most individuals, especially if admission to hospital is required. Risk of relapse needs to inform discussions about treatment.

\section{Cognitive decline}

The issue of cognitive decline associated with first-episode psychosis deserves particular attention. Lewandowski et al (2011) provide a useful overview and highlight the difference between schizophrenia and bipolar disorder. In schizophrenia, the biggest changes occur during and immediately after the first psychotic episode. In bipolar disorder, each successive relapse leads to further deterioration in cognitive functioning. For most individuals with first-episode psychosis the diagnosis and pattern of future cognitive problems will be unclear. However, a key question will be the likely effect of antipsychotic medication on mental abilities. There is evidence that effective treatment of positive symptoms using antipsychotic medication can improve cognitive functioning in schizophrenia and first-episode psychosis (Keefe 2004). However, a recent meta-analysis indicates that antipsychotics may cause structural brain change (Fusar-Poli 2013). The relationship between structural brain changes and actual cognitive decline requires further exploration (Leeson 2011). Of note in this regard is a small study involving 42 patients with first-episode psychosis which indicated that antipsychotic discontinuation or dose reduction led to improved neurocognition (Faber 2011).

\section{Problems with maintenance medication}

The potentially serious short- and longer-term medical complications and other side-effects associated with antipsychotic treatment are now well recognised (Foley 2011). Young adults and adolescents are more likely to experience extrapyramidal side-effects even when taking second-generation antipsychotics (Correll 2008). Metabolic disturbances also seem to be more likely in younger individuals (Correll 2008). Clinically significant antipsychotic-induced hyperprolactinaemia can cause hypogonadism. This in turn will adversely affect the important bone mineralisation that occurs during the teens and 20 s.

In response to the range of adverse effects there is now widespread advice and guidelines aimed at harm minimisation. Monitoring of metabolic and cardiovascular parameters for individuals before the start of and during antipsychotic treatment are basic requirements. However, repeated audits of clinicians' adherence to monitoring guidelines for patients on antipsychotics demonstrate inconsistent outcomes (Foley 2011). Logistical challenges in carrying out regular blood tests and physical health checks in community settings may account for some of the difficulties. Furthermore, it is not clear what effect monitoring regimes have on the decisions of patients and their families to continue with maintenance medication. Some individuals will find the monitoring procedures reassuring, whereas for others they will be a deterrent to continuing treatment. The regular blood tests may be experienced as inconvenient and 
unpleasant, and they can contribute to feelings of stigma and raise understandable anxieties about possible health problems in later life. Relapse of psychosis on discontinuing medication may seem an acceptable risk if it means avoidance of serious medication side-effects.

Thus, we can see how the balancing of probabilities for individual patients is an imprecise task. There is lack of certainty about who is at risk, both of psychotic relapse and medication side-effects, and when. Our evidence base concerning first-episode psychosis is hampered by inconsistent study methods. Comparing different studies is problematic because of a long list of factors. These include not only variability in non-medical treatments, service organisation, definitions and categorisations of patient samples and outcomes, but also confounding factors such as substance misuse (Marshall 2011). Even what we think we know about risks and benefits of maintenance medication relates to groups of patients. Predictions of outcomes for specific individuals are therefore unreliable.

It could be argued that degrees of uncertainty are a feature of all prognostic predictions in psychiatry and medicine as a whole. This may be a source of anxiety for clinicians and patients alike, particularly when possible adverse outcomes are serious. A return to the evidence concerning informed decision-making, psychoeducation and doctors' communication skills will help to guide clinical practice in the face of ambiguity.

\section{Engaging patients in decision-making regarding medication}

\section{Psychoeducation}

The above discussion about risks and benefits of maintenance medication following firstepisode psychosis contains the assumption that decision-making will be based on consideration of the evidence. Doctors are expected to have a good knowledge of the scientific evidence base underpinning their practice. Thus, this article, which is primarily addressed to doctors, needs to start with the same. However, many human decisions are influenced by emotional factors or deeply held core beliefs rather than facts alone (Miklowitz 2010). This might explain why many psychoeducational strategies for patients are seen as poorly effective (Lincoln 2007).

Psychoeducation has been variously described. In their meta-analysis of psychoeducation in psychotic disorders, Lincoln et al (2007) define it as 'a systematic didactic psychotherapeutic intervention designed to inform patients and their families about the disorder and coping' They report that psychoeducation directed at patients alone had minimal effect on symptoms, medication adherence or functioning, although knowledge about their illness was improved. Psychoeducation involving families, however, was more effective.

\section{Applying psychological models of medication adherence}

Low levels of medication adherence have been explored for a range of medical conditions. In mental health settings, specific measures such as depot injections of long-acting antipsychotic preparations may be helpful in improving adherence for those wishing to reduce the risk of relapse of psychosis (National Institute for Health and Care Excellence 2014). It is also helpful to review the various explanatory models that break down decision-making about medication into components. A number of psychological approaches have arisen from these models, including adherence therapy (see below) and cognitive-behavioural therapy (CBT).

Alongside these specific approaches that aim to improve medication adherence, associated developments include the promotion of shared decision-making and motivational interviewing techniques. One example is the Making Good Decisions in Collaboration (MAGIC) programme commissioned by the Health Foundation. In a recent paper, Mulley and colleagues (2012) describe a stepped approach based on the MAGIC programme which aims to identify patients' priorities and help doctors to adequately inform patients about treatment options. The authors suggest strategies that can be taken directly into clinical consultations. One such is 'a mindset of scientific detachment', which helps doctors avoid misleading questions such as 'What advice would I give to my relatives?'. The authors remind us that such professional self-talk may reflect the clinician's rather than the patient's values. They also address the important question regarding at what point doctors should make specific recommendations, and also how to assess whether patients are making decisions which are inconsistent with their own priorities. The implication of the paper is that doctors can bring strategies to facilitate informed decision-making into everyday clinical practice.

\section{Shared decision-making}

All doctors and medical students are expected to learn and refresh their communication skills as a core competency. This includes developing 
self-awareness and internal supervision. A patient-centred approach to shared decisionmaking is recommended in NICE guidelines on schizophrenia (National Institute for Health and Clinical Excellence 2013; National Institute for Health and Care Excellence 2014) and outlined in detail in a Royal College of Psychiatrists' CPD Online module (Chaplin 2012). This module also acknowledges the inherent contradiction for many psychiatrists: we endorse patient-centred informed decision-making, but on occasion use the Mental Health Act to administer compulsory treatment. If patients are becoming very unwell and their decision-making capacity is seriously impaired the power balance shifts in consultations. Communication can become uncomfortable and adversarial. This is the particular feature of many psychiatric consultations that mitigates against a mindset of either scientific or emotional detachment. Applying the MAGIC-based model to psychiatric practice (Mulley 2012) reveals the importance of considering both positive and negative adverse outcomes related to medication choices at appropriate stages of illness. That is, medication options for an episode of acute illness can be discussed while the patient is still well, so that their preferences are known should they later become ill.

Psychiatrists and other clinicians working in specialist first-episode psychosis services have an important role in exploring patients' opinions on two particular matters. First, what a good outcome is and how to achieve it, and second, how to minimise and prepare for negative outcomes, including relapse. Specific training packages and tools may facilitate this process and will be considered below. Clinicians will need to be particularly aware of how cultural and religious factors can affect decision-making. An appreciation of the changing nature of scientific knowledge relating to this area is needed. Also, an understanding of decision-making processes specifically in young people will be required. These will also be considered below.

\section{Adherence therapy}

Adherence therapy seeks to address the complex range of factors influencing medication use. It has been variously described, but essentially it is a patient-centred approach that examines the individual's beliefs and involves goal-setting and problem-solving (Brown 2013). Evidence of its long-term effect on adherence to antipsychotic treatment is variable (Abdel-Baki 2012). In the randomised controlled trial of relapse prevention therapy for first-episode psychosis by Gleeson et al (2009), the package of treatment under consideration approximates to adherence therapy and results were positive.

A small study examined the effectiveness of a specific package of adherence therapy delivered by an EIP team after 6 days of training (Brown 2013). The qualitative results suggest that patients approved of the collaborative approach and used the opportunities to weigh up the benefits of medication. The clinicians who participated in the study expressed positive views, but noted the large amount of time involved in the training and practice. This type of small study is less well suited to demonstrating 'hard' outcomes concerning levels of medication use or relapse rates. However, the study tells us about degrees of satisfaction and engagement following adherence therapy for a young sample (mean age 25 years).

The study by Brown et al (2013) also shows some of the difficulties front-line clinicians face when incorporating manualised or structured treatments into their everyday clinical work. Mounting administration tasks and the need to respond to crises mitigate against the delivery of regular, structured treatments. White (2013) argues that pharmacists may be particularly well placed to introduce CBT and adherence therapy principles into discussions with patients in order to improve concordance. Patients may see pharmacists as more removed from the everyday responsibilities of care and risk management, and consider them a more independent source of information and support.

\section{Motivational interviewing}

Motivational interviewing techniques, initially introduced into the field of addictions, aim to change established behaviours and introduce new ones. They can be used in a variety of mental health settings (Treasure 2013), including medication adherence. There is some overlap with components of adherence therapies and shared decision-making. However, motivational interviewing is even more patient centred than these approaches and is only suitable for those fully competent to make decisions. The patient is regarded as an expert and the therapist adopts a 'lower power position' while using differential reinforcement to promote 'change talk'. The approach represents a significant shift from the psychiatrist's usual expert role, and may be difficult to apply in full. However, the Royal College of Psychiatrists' CPD Online module (Treasure 2013) demonstrates the effectiveness of specific strategies. 


\section{Guided discontinuation, relapse plans and non-medical prescribing}

If maintenance medication is not agreed, alternative strategies can be developed in keeping with the principles of shared and informed decisionmaking (Box 3) (National Institute for Health and Clinical Excellence 2013; National Institute for Health and Care Excellence 2014). Gradually reducing the dose of antipsychotic with a view to cessation can be combined with a clear relapse plan (Abdel-Baki 2012). This approach is not well supported by evidence, but is familiar to clinicians. It involves identifying early warning signs, and when and how to re-establish medication. Such plans rely on engagement with prescribers and access to medication. Non-medical prescribers may be more accessible during relapse and provide a useful clinician adjunct. The efficiency and cost-effectiveness of non-medical prescribers in this area needs further study.

Despite widespread use of crisis relapse plans for those with more established mental illness, their effectiveness has not been supported (Thornicroft 2013). Nevertheless, the case for collaborative relapse planning is even more powerful for those who do not agree to maintenance antipsychotic treatment. Alternative approaches, including compulsory community-based treatment under community treatment orders (CTOs), are not supported by a recent study (Burns 2013), although further research into the usefulness of CTOs seems indicated, given their widespread use and popularity with individual clinicians.

\section{Considerations when working with adolescents and young adults}

\section{Cognitive development}

In considering how young people may reason and think about mental illness and treatment, it is useful to return to the theories of Jean Piaget developed during the 1950s. Piaget and others

BOX 3 Possible antipsychotic management strategies following recovery from first-episode psychosis

- Maintenance medication: continuing the therapeutic dose even after symptoms have receded

- Guided discontinuation: reducing the dose until cessation of medication under medical supervision

- Intermittent symptom-targeted medication: administering an antipsychotic as required, according to symptom and/or other criteria described how 'formal operational thinking' can be expected to develop during puberty (Coleman 2011). Formal operational thought involves hypothesis-testing and abstract thinking, and it can be demonstrated in experimental problemsolving situations.

Piaget's theories have subsequently been revised and criticised, but their essence remains relevant. We now know that many individuals do not acquire formal operational thinking capabilities in a predictable manner, and the developmental process is variable and may be delayed into adulthood (Coleman 2011). Also, environmental factors, including educational opportunities, are influential.

Our understanding of cognitive development has become more sophisticated with the expansion of neuroscience specialties over recent years. Blakemore \& Choudhury (2006) note that structural brain changes continue well beyond adolescence and into the late 20 s. Crone et al (2008), among others, have proposed neurophysiological models to explain the observed shifts away from emotional, impulsive decisionmaking towards more rational approaches during adolescence. Brain system theories also highlight how young people's abilities to incorporate the views of others concerning risk improve with age. These neurocognitive models indicate that tasks such as planning life goals may be affected by a tendency to respond to immediate rewards and emotions in preference to considering future risks.

Clearly, these issues will affect care planning for young people recovering from first-episode psychosis. Their consideration of the debates concerning maintenance medication will be influenced by their incompletely developed reasoning. In addition, a psychotic episode is likely to interrupt social, educational, emotional and cognitive development. This disruption may further delay maturation at exactly the time when affected individuals are being asked to adjust to the onset of major difficulties and make complex choices about treatment. Any cognitive impairment that occurs leading up to, during or after a first episode of psychosis will compound the situation (Lewandowski 2011). Psychiatrists working with adults with more established mental illness, including schizophrenia, are aware of how executive functioning, memory and processing speed problems affect decision-making capacity.

\section{Social development and families}

The above considerations highlight the need for young people with first-episode psychosis to be supported by their parents and other trusted 
adults, including mental health professionals, when making decisions about treatment. The importance of involving families in psychoeducation is also recognised (Lincoln 2007). Adolescents and young adults are increasingly influenced by peers as they grow older. Nevertheless, research into this area has consistently shown that parents remain influential and an important source of advice (Graham 2004).

Miklowitz (2010) has researched and described family-focused approaches to treatments for individuals, including adolescents, with bipolar disorder. The author provides a useful stepped approach to addressing medication non-adherence within family interviews. Of particular note is the need to clarify the symbolic significance of nonadherence. For example, non-adherence can be interpreted as an expression of a young person's quest for autonomy within a family. Much of this detail about family interventions is likely to be applicable to young people with first-episode psychosis. Where relationships with parents are fragmented, other sources of adult support and mentoring will be needed.

\section{Adolescent identity and relationships with professionals}

Several other developmental aspects associated with adolescence and young adulthood will affect how individuals respond to the onset of mental illness, recovery and treatment. The emerging sense of an adult self and identity will be disrupted by the arrival of mental illness. This change in identity was explored in the study published by EDEN group mentioned earlier (Lester 2011). The young participants highlighted their experiences of a changed sense of self and of perceived stigma. Changes in appearance, including weight gain due to medication side-effects, were contributors in both these areas. The anticipated autonomy and separation from parents and important adults is also affected. A young person's resistance to the implications of first-episode psychosis is likely to be a reflection of these kinds of issue.

As regards the planning of medication treatment, clinicians might expect to encounter questioning and resistance to advice. However, such challenges can be seen as a positive sign of maturation. Debate is healthy and it frees up clinicians to pose questions about reasons for non-adherence. It is also in keeping with the principles regarding patient-centred decisionmaking outlined in this article. A Socratic dialogue which encourages the patient to think through their decisions is one technique that can be usefully employed (Box 4).
BOX 4 Medication adherence: clinical scenarios

Typical approach

The clinician asserts their knowledge and status: 'If you take this medication I believe it will help you stay out of hospital'

\section{Alternative approach}

Socratic questioning: 'Some patients who have experienced psychosis stay on medication after the symptoms have gone. You might ask why would they go through all the bother and side-effects of taking medication. Would it be because their doctor advises this? Or would it be because they believe it will help them stay out of hospital?'

This leads to patient-centred decision-making and goal-setting: 'Can we talk about what are going to be the most important things for you over the next few years? For example, how important is it for you to stay out of hospital? What do you think will help you achieve your goals?'

\section{Conclusions}

Following a first episode of psychosis there will be many questions as to how well and in what way a young person can engage in decision-making about their treatment. Thanks to the availability and specific characteristics of EIP services, these questions can be explored over time and in an ageappropriate way. Psychiatrists have a central role and particular skills to offer in this process: first, in making sense of the latest evidence concerning medication strategies; second, in applying knowledge of the developmental processes affecting and arising from mental illness; and third, in applying their extensive training in communication and interviewing skills. These are also areas where further research is needed (Box 5).

BOX 5 Areas for future research regarding medication management following recovery from first-episode psychosis

- Identifying which clinical and other characteristics predict a higher rate of relapse

- Better understanding of young people's views about antipsychotic treatment strategies for reducing the risk of relapse

- Establishing the most effective interventions to help young people and their families manage medication 
A developmental perspective highlights how psychiatrists can facilitate the transition to the adult arena, where complex decision-making tasks will increasingly dominate. The exploration of evidence and decision-making concerning medication maintenance is a paradigm of one of the major developmental tasks of adolescence and young adulthood for those with first-episode psychosis.

\section{References}

Abdel-Baki A, Ouellet-Plamondon C, Malla A (2012) Pharmacotherapy challenges in patients with first episode psychosis. Journal of Affective Disorders, 138: s3-14.

Alvarez-Jimenez M, Parker AG, Hetrick SE, et al (2009) Preventing the second episode: a systematic review and meta-analysis of psychosocial and pharmacological trials in first episode psychosis. Schizophrenia Bulletin, 37: 619-30.

Blakemore SJ, Choudhury S (2006) Development of the adolescent brain: implications for executive function and social cognition. Journal of Child Psychology and Psychiatry, 47: 296-312.

Brown E, Gray R, James M, et al (2013) Effectiveness of adherence therapy in patients with early psychosis: a mirror image study. International Journal of Mental Health Nursing, 22: 24-34.

Burns T, Rugkåsa J, Molodynski A, et al (2013) Community treatment orders for patients with psychosis (OCTET): a randomised controlled trial. Lancet, 381: 1627-33.

Chaplin R, Quirk A (2012) How patient-centred are you? Shared decision-making in psychiatric practice (learning module). Royal College of Psychiatrists CPD Online (http://www.psychiatrycpd.co.uk/ learningmodules/howpatient-centredareyous.aspx).

Chen EYH, Christy LMH, May MLL, et al (2010) Maintenance treatment with quetiapine versus discontinuation after one year treatment in patients with remitted first episode psychosis: a randomised controlled trial. BMJ, 341: c4024.

Coleman JC (2011) The Nature of Adolescence (4th edn). Routledge.

Correll CU (2008) Monitoring and management of antipsychoticrelated metabolic and endocrine adverse events in pediatric patients. International Review of Psychiatry, 20: 195-201.

Crone EA, Bullens L, Van Der Plas EAA, et al (2008) Developmental changes and individual differences in risk taking and perspective taking in adolescence. Developmental Psychopathology, 20: 1213-29.

Faber G, Smid HG, Van Gool A, et al (2011) The effects of guided discontinuation of antipsychotics on neurocognition in first episode psychosis. European Psychiatry, 27: 275-80.

Foley DL, Moreley K (2011) Systematic review of early cardiometabolic outcomes of the first treated episode of psychosis. Archives of General Psychiatry, 68: 609-16.

Fusar-Poli P, Smieskova R, Kempton MJ, et al (2013) Progressive brain changes in schizophrenia related to antipsychotic treatment? A metaanalysis of longitudinal MRI studies. Neuroscience and Biobehavioral Reviews, 37: 1680-91.

Gleeson JFM, Cotton S, Alvarez-Jimenez M, et al (2009) A randomised controlled trial of relapse prevention therapy for first-episode psychosis patients. Journal of Clinical Psychiatry, 70: 477-86.

Graham P (2004) The End of Adolescence. Oxford University Press.

Gulliver G, Griffiths K, Christensen H (2010) Perceived barriers and facilitators to mental health help-seeking in young people: a systematic review. BMC Psychiatry, 10: 113.

Jones PB (2013) Adult mental health disorders and their age at onset. British Journal of Psychiatry, 202 (suppl 54): s5-10.

Josiassen RC, Shaughnessy RA, Filymer DM, et al (2010) Early intervention with second-generation antipsychotics in first-episode psychosis: results of an 8-week naturalistic study. Journal of Early Intervention in Psychiatry, 4: 57-63.

Keefe RSE, Seidman LJ, Christensen BK, et al (2004) Comparative effect of atypical and conventional antipsychotic drugs on neurocognition in first-episode psychosis: a randomized, double-blind trial of olanzapine versus low doses of haloperidol. American Journal of Psychiatry, 161: 985-95

Kennedy E, Kumar A, Datta SS (2007) Antipsychotic medication for childhood-onset schizophrenia. Cochrane Database of Systematic Reviews, 3: CD004027.

Leeson VC, Sharma P, Harrison M, et al (2011) IO trajectory, cognitive reserve, and clinical outcome following a first episode of psychosis: a 3-year longitudinal study. Schizophrenia Bulletin, 37: 768-77.

Lennox BR, Coles AJ, Vincent A (2012) Antibody-mediated encephalitis: a treatable cause of schizophrenia. British Journal of Psychiatry, 200: $92-4$

Lester H, Khan N, Jones P, et al (2011) Service users' views of moving on from early intervention services for psychosis: a longitudinal qualitative study in primary care. Psychiatric Services, 62: 882-7.

Leucht S, Tardy M, Komossa K, et al (2012) Antipsychotic drugs versus placebo for relapse prevention in schizophrenia: a systematic review and meta-analysis. Lancet, 379: 2063-71.

Lewandowski EKE, Cohen BM, Ongur D (2011) Evolution of neuropsychological dysfunction during the course of schizophrenia and bipolar disorder. Psychological Medicine, 2: 225-41.

Lincoln TM, Wilhelm K, Nestoriuc Y, et al (2007) Effectiveness of psychoeducation for relapse, symptoms, knowledge, adherence and functioning in psychotic disorders: a meta-analysis. Schizophrenia Research, 96: 232-45.

Marshall M, Rathbone J (2011) Early intervention for psychosis. Cochrane Database of Systematic Reviews, 6: CD004718.

McGorry P, Alvarez-Jimenez M, Killackey E (2013) Antipsychotic medication during the critical period following remission from first episode psychosis: less is more. JAMA Psychiatry, 70: 898-900.

Miklowitz D (2010) Bipolar Disorder: A Family-Focused Treatment Approach. Guilford Press.

Mulley A, Trimble C, Elwyn G (2012) Stop the silent misdiagnosis: patients' preferences matter. BMJ, 345: e6572.

National Institute for Health and Clinical Excellence (2013) Psychosis and Schizophrenia in Children and Young People: Recognition and Management (NICE Clinical Guideline 155). NICE.

National Institute for Health and Care Excellence (2014) Psychosis and Schizophrenia in Adults: Treatment and Management (NICE Clinical Guideline 178). NICE.

Robinson D, Waerner MG, Alvir JM, et al (1999) Predictors of relapse following response from a first episode of schizophrenia or schizoaffective disorder. Archives of General Psychiatry, 56: 241-7.

Thornicroft G, Rugkåsa J, Molodynski A, et al (2013) Clinical outcomes of joint crisis plans to reduce compulsory treatment for people with psychosis: a randomised controlled trial. Lancet, 381: 1627-33.

Treasure J, Macdonald P (2013) Introduction to motivational interviewing (learning module). Royal College of Psychiatrists CPD Online (http://www.psychiatrycpd.org/learningmodules/ introductiontomotivational.aspx)

White D (2013) Discuss patients' perceptions of treatment to tackle non-adherence. Clinical Pharmacist, 5: 85-6.

World Health Organization (1992) International Classification of Mental and Behavioural Disorders, Tenth Edition (ICD-10). WHO.

Wunderink L, Nieboer RM, Wiersma D, et al (2013) Recovery in remitted first-episode psychosis at 7 years of follow-up of an early dose reduction/discontinuation or maintenance treatment strategy: long-term follow-up of a 2-year randomized clinical trial. JAMA Psychiatry, 70: 913-20.

\section{MCO answers \\ 1 a 2 a 3 e $4 d \quad 5 c$}




\section{MCQs}

Select the single best option for each question stem

1 As regards medication adherence in young people:

a parental advice can be influential

b it is best to avoid considering identity issues as part of the discussion

c younger individuals are not able to consider the full implications of medication non-adherence

$d$ the reasons for non-adherence are usually obvious

e providing too much detailed information about side-effects will reduce adherence rates.

2 In young people recovering from first-episode psychosis, maintenance antipsychotic medication for 1-2 years:

a reduces risk of relapse for most individuals

b should not be considered unless a firm diagnosis of schizophrenia has been made c is of limited benefit if psychosocial treatments are available

$d$ is the most important part of the care plan

e may raise concerns about physical health sideeffects, but these never outweigh the benefits of relapse prevention.

3 The effects of maintenance antipsychotics on neurocognitive functioning:

a are unrelated to number of relapses

b are not evident immediately

c are easy to predict

d will not need to be assessed unless there is a complaint of drowsiness

e are hard to differentiate from other causes of neurocognitive impairment.

4 As regards first-episode psychosis:

a clinical characteristics reliably predict which individuals can discontinue antipsychotic medication on remission b monitoring of calorie intake need not occur until antipsychotic treatment is well established

c most antipsychotics are licensed for the treatment of first-episode psychosis

d young people with first-episode psychosis need to identify for themselves what is a positive outcome of treatment

e community treatment orders are likely to be helpful at this stage of illness.

5 Young people with psychosis:

a are likely to seek help at an early stage

b are no more likely to engage with early intervention services than with generic mental health services

c are more sensitive to medication side-effects

d are unlikely to consider the effects of stigma

e can be given a firm diagnosis at first presentation. 